\title{
A Comparison Study of Modelling Techniques for Permanent Magnet Machines
}

\author{
Kesavan Ramakrishnan ${ }^{1}$, Mitrofan Curti $^{2}$, Damir Zarko ${ }^{3}$, Gianpiero Mastinu ${ }^{1}$ \\ Johannes J.H. Paulides ${ }^{2}$ and Elena A. Lomonova ${ }^{2}$ \\ ${ }^{1}$ Department of Mechanical Engineering, Politecnico di Milano, Piazza Leonardo da Vinci 32, 20133 Milan, Italy \\ ${ }^{2}$ Eindhoven University of Technology, Eindhoven, 5600 MB, The Netherlands \\ ${ }^{3}$ University of Zagreb Faculty of Electrical Engineering and Computing, \\ Department of Electrical Machines, Drives and Automation \\ Email:kesavan.ramakrishnan@polimi.it, M.Curti@tue.nl,damir.zarko@fer.hr
}

\begin{abstract}
In this paper, four different modelling techniques for permanent magnet (PM) machines are compared for their accuracy and computational complexity. The considered techniques are primarily based on conformal mapping and harmonic modelling. In conformal mapping, the slotted air gap is mapped into a simpler canonical shape, where the field solution is calculated and then mapped back to the original domain. In harmonic modelling, the regions of the machine cross section are represented as Fourier series and coupled with each other by means of boundary conditions. The field solution is obtained by solving the boundary value problem. In order to quantify the accuracy of the field solutions, global parameters such as cogging torque and flux linkage are computed. The effectiveness of the modelling techniques are evaluated by comparing the global parameters and the simulation time with finite element analysis (FEA) results.
\end{abstract}

Index Terms-Harmonic Modelling, Relative Permeance, Complex relative Permeance, Conformal Mapping, SchwarzChristoffel Toolbox, FEA

\section{NOMENCLATURE}

$B_{r} \quad$ Magnet remanence (T)

$B_{s l r} \quad$ Slotless air-gap radial flux density $\left(\mathrm{Wb} / \mathrm{m}^{2}\right)$

$B_{s l \theta} \quad$ Slotless air-gap tangential flux density

$B_{s} \quad$ Slotted air-gap flux density $\left(\mathrm{Wb} / \mathrm{m}^{2}\right)$

$B_{s \theta} \quad$ slotted air gap tangential flux density

$J_{z}$

$H$

$A_{z}$

$\tilde{\lambda}$

$\lambda$

$\mu_{0}$

$\mu_{r}$

$p$

$\alpha_{p}$

$Q s$

$\operatorname{lm}$

$g$

$R_{g}$

$R_{r}$

$R_{m}$

$R_{s}$

\author{
$l_{a} \quad$ stack length (m) \\ $b_{0} \quad$ Slot opening (m) \\ $d_{s} \quad$ Slot depth (m) \\ $N_{c} \quad$ No. of conductors in a slot \\ $T_{c} \quad$ Cogging Torque $(\mathrm{Nm})$ \\ $r, \theta \quad$ Coordinates of air-gap evaluation points
}

\section{INTRODUCTION}

Permanent Magnet (PM) machines are widely used for their high efficiency and power density. The motor requirements on volume, axial length, outer diameter, efficiency, weight and cost are different [1], [2] for each of these applications. Optimal selection of the motor parameters is essential to meet these requirements effectively. In order to use a motor model in an optimization routine, it has to be accurate and computationally cheap. There are several modelling techniques available in literature, which have their own merits and demerits. In this study, four such models are analyzed and the results are compared with FEM.

In the mathematical models, for simplicity, the end windings are neglected, the iron is assumed to have infinite permeability, and the magnet end effects are not considered. With these assumptions, the electro-magnetic problem can be solved for the magnetic vector or scalar potential in two dimension and the Laplacian or Poissonian equations are solved for the field solutions [3]-[6]. The field solutions can further be used to calculate torque, back-emf, and losses.

The modelling techniques considered in this study are relative permeance model (RP) [7], complex permeance model (CP) [8], Schwarz-Christoffel Toolbox model (SC) [9], and Harmonic model(HM) [5]. The first three models are fundamentally based on conformal mapping. For the SC toolbox model, MATLAB toolbox, which allows numerical mapping of complex polygons to simple rectangles, can be utilized. The harmonic model allows one to use the exact solutions of Laplacian or Poissonian equations that are represented as Fourier series in the tangential direction [10], [11]. 


\section{Modelling TECHNiQues}

A twenty pole permanent magnet machine is considered in the analysis as a test bench, shown in Fig.1, and its parameters are given in Table I.

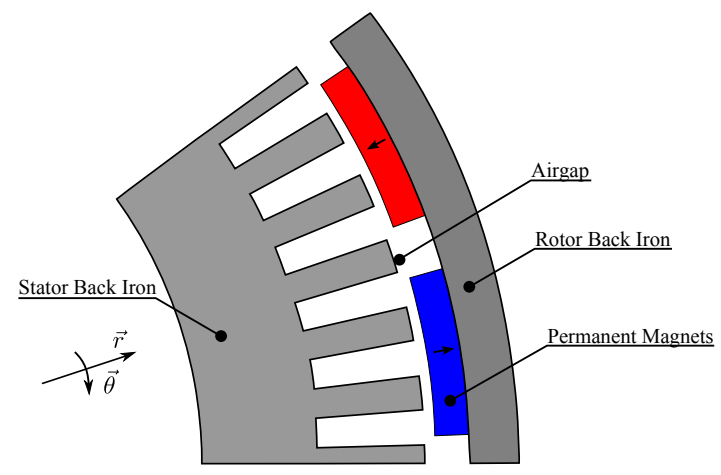

Fig. 1. Test bench machine for the comparison of modelling techniques

TABLE I

Parameters of External Rotor Surface PM Motor

\begin{tabular}{cccc}
\hline Parameters, & Symbol, & Value, & Unit \\
\hline Number of poles & $2 p$ & 20 & - \\
Slot number & $Q_{s}$ & 60 & - \\
Magnet arc/pole pitch ratio & $\alpha_{p}$ & 0.75 & - \\
Air gap length & $g$ & 1.2 & $\mathrm{~mm}$ \\
Radius of the rotor surface & $R_{r}$ & 85 & $\mathrm{~mm}$ \\
Radius of the magnet surface & $R_{m}$ & 76.2 & $\mathrm{~mm}$ \\
Stator outer radius & $R_{s}$ & 75 & $\mathrm{~mm}$ \\
Magnet remanence & $B_{r}$ & 1.19 & $\mathrm{~T}$ \\
Core length & $l_{a}$ & 60 & $\mathrm{~mm}$ \\
Slot depth & $d_{s}$ & 12 & $\mathrm{~mm}$ \\
No. of conductors in a slot & $N_{c}$ & 6 & - \\
\hline
\end{tabular}

\section{A. Relative permeance model}

The radial and tangential components of the slotless air gap field solution are obtained in the polar coordinates using (1) to (4). In order to capture the slotting effect, the radial field solution is multiplied with relative permeance. The influence of slotting on tangential component of the air gap field is neglected.

The slotless air gap field solution given in [12]:

When $(n p \neq 1)$

$$
\begin{gathered}
B_{s l r}=\sum_{n=1,3 . .}^{\infty} \frac{-4 n p B_{r} \sin \frac{n \pi \alpha_{p}}{2}}{n \pi \mu_{r}\left((n p)^{2}-1\right)}\left[\left(\frac{r}{R_{m}}\right)^{n p-1}+\left(\frac{R s}{R_{m}}\right)^{n p-1}\left(\frac{R s}{r}\right)^{n p+1}\right] \\
\left\{\frac{(n p-1)\left(\frac{R m}{R r}\right)^{2 n p}+2\left(\frac{R m}{R r}\right)^{n p-1}-(n p+1)}{\frac{\mu_{r}+1}{\mu_{r}}\left[1-\left(\frac{R s}{R r}\right)^{2 n p}\right]-\frac{\mu_{r}-1}{\mu_{r}}\left[\left(\frac{R s}{R m}\right)^{2 n p}-\left(\frac{R m}{R r}\right)^{2 n p}\right]} \cos (n p \theta)\right. \\
B_{s l \theta}=\sum_{n=1,3 . .}^{\infty} \frac{-4 n p B_{r} \sin \frac{n \pi \alpha_{p}}{2}}{n \pi \mu_{r}\left((n p)^{2}-1\right)}\left[\left(\frac{-r}{R_{m}}\right)^{n p-1}+\left(\frac{R s}{R_{m}}\right)^{n p-1}\left(\frac{R s}{r}\right)^{n p+1}\right] \\
\left\{\begin{array}{c}
(n p-1)\left(\frac{R m}{R r}\right)^{2 n p}+2\left(\frac{R m}{R r}\right)^{n p-1}-(n p+1) \\
\frac{\mu_{r}+1}{\mu_{r}}\left[1-\left(\frac{R s}{R r}\right)^{2 n p}\right]-\frac{\mu_{r}-1}{\mu_{r}}\left[\left(\frac{R s}{R m}\right)^{2 n p}-\left(\frac{R m}{R r}\right)^{2 n p}\right]
\end{array}\right\} \sin (n p \theta)
\end{gathered}
$$

and when $(n p=1)$

$$
\begin{aligned}
& B_{s l r}=\sum_{n=1,3 . .}^{\infty} \frac{2 n p B_{r} \sin \frac{n \pi \alpha_{p}}{2}}{n \pi \mu_{r}\left((n p)^{2}-1\right)}\left[1+\left(\frac{R s}{r}\right)^{2}\right] \\
& \left\{\frac{n p\left(\frac{R m}{R s}\right)^{2}-n p\left(\frac{R r}{R s}\right)^{2}+\left(\frac{R r}{R s}\right)^{2} \ln \left(\frac{R m}{R r}\right)^{2}}{\frac{\mu_{r}+1}{\mu_{r}}\left[1-\left(\frac{R r}{R s}\right)^{2}\right]-\frac{\mu_{r}-1}{\mu_{r}}\left[\left(\frac{R m}{R r}\right)^{2}-\left(\frac{R r}{R m}\right)^{2}\right]}\right\} \cos (n p \theta) \\
& B_{s l \theta}=\sum_{n=1,3 . .}^{\infty} \frac{2 n p B_{r} \sin \frac{n \pi \alpha_{p}}{2}}{n \pi \mu_{r}\left((n p)^{2}-1\right)}\left[-1+\left(\frac{R s}{r}\right)^{2}\right] \\
& \left\{\frac{n p\left(\frac{R m}{R s}\right)^{2}-n p\left(\frac{R r}{R s}\right)^{2}+\left(\frac{R r}{R s}\right)^{2} \ln \left(\frac{R m}{R r}\right)^{2}}{\frac{\mu_{r}+1}{\mu_{r}}\left[1-\left(\frac{R r}{R s}\right)^{2}\right]-\frac{\mu_{r}-1}{\mu_{r}}\left[\left(\frac{R m}{R r}\right)^{2}-\left(\frac{R r}{R m}\right)^{2}\right]}\right\} \sin (n p \theta)
\end{aligned}
$$

The relative permeance $(\tilde{\lambda})$ is derived as a function of flux path length and modification factor $(\gamma)$ [7]. The radial variation of the field solution can be captured by varying the modification factor $(\gamma)$ that changes the effective flux path length as shown in Fig.2.

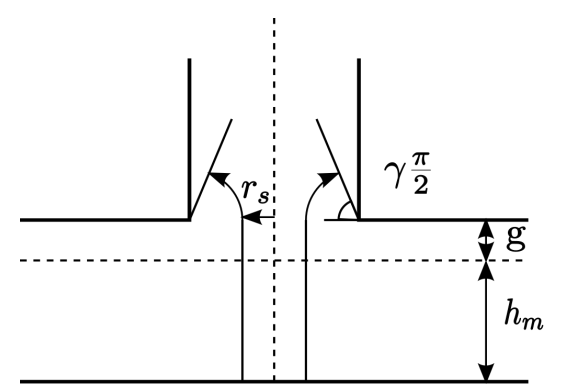

Fig. 2. Relative permeance model

So the relative permeance can be expressed as,

$\tilde{\lambda}= \begin{cases}\frac{g+l_{m} / \mu_{R}}{g+l_{m} / \mu_{R}+\gamma \frac{\pi}{2}\left[\frac{b_{0}}{2}-r_{s}\right]}, & \text { for region of the slot opening } \\ 1, & \text { for region of the tooth }\end{cases}$

where,

$$
\gamma=\frac{g+l_{m} / \mu_{R}}{\pi b_{0} / 4}\left(\frac{B_{\max }}{B_{\min }}-1\right)
$$

The cogging torque $\left(T_{c}\right)$ is calculated by summing the lateral forces acting on the teeth as in [7]:

$$
T_{c}=\sum_{k=1}^{Q s} l a \int_{0}^{\frac{b_{0}}{2}}\left(\frac{B_{1}^{2}-B_{2}^{2}}{2 \mu_{0}}\right) r_{t} d y
$$

where $b_{0}$ is slot opening, $r_{t}$ is equal to $R_{s}-r_{s}$, and $B_{1}$ and $B_{2}$ are the flux densities along opposite sides of the slot walls.

\section{B. Complex relative permeance model}

The relative permeance model fairly well estimates the radial component of the field solution in the slotted air gap, but it does not include the tangential component which can be useful to derive the closed form solution for the cogging torque and electromagnetic torque based on integration of 
the Maxwell's stress tensor. The real and imaginary parts of the complex relative permeance are derived to take into account the influence of slotting on both radial and tangential components of the air gap flux density. The field distribution in the slotted air gap $\left(B_{s}\right)$ is obtained by multiplying the slotless air gap flux density $\left(B_{s}\right)$ and the complex conjugate of the relative permeance $\left(\lambda^{*}\right)$.

$$
B_{s}=B_{k} \lambda^{*}=B_{k}\left(\frac{\partial k}{\partial s}\right)^{*}=B_{k}\left(\frac{\partial k}{\partial t} \frac{\partial t}{\partial w} \frac{\partial w}{\partial z} \frac{\partial z}{\partial s}\right)^{*}
$$

Each partial derivative in (7) is defined by conformal transformation explained in [8] and the final expression is

$$
B_{s}=B_{k}\left[\frac{k}{s} \frac{(w-1)}{(w-a)^{\frac{1}{2}}(w-b)^{\frac{1}{2}}}\right]
$$

so the complex relative permeance is written as

$$
\lambda=\lambda_{a}+j \lambda_{b}=\frac{k}{s} \frac{(w-1)}{(w-a)^{\frac{1}{2}}(w-b)^{\frac{1}{2}}}
$$

The radial and tangential components of the slotted air gap field solution are derived as

$$
\begin{aligned}
& B_{s}=B_{s r}+j B_{s \theta}=B_{k} \lambda^{*}= \\
&\left(B_{r} \lambda_{a}+B_{\theta} \lambda_{b}\right)+j\left(B_{\theta} \lambda_{a}+B_{r} \lambda_{b}\right) .
\end{aligned}
$$

In order to employ equation for the slotless air gap field solution [12], the mapped evaluation points are assumed to form a circular arc and the magnets are considered to retain their shapes. Although these two assumptions allow one to derive the closed form solution for flux density and cogging torque, they will impair the accuracy [8].

\section{Schwarz-Christoffel Toolbox}

The field solution can be calculated with better accuracy by capturing the distortions of the magnet shape and evaluation points in the slotless domain. For this purpose, the numerical conformal mapping using SC Toolbox [9] is employed and Hague's [3] equation is solved.

The canonical domain field solution is given as [3]

$$
\begin{array}{r}
\Omega=\frac{\mu_{0} I}{2 \pi}\left(\arctan \left[\frac{\tan \left(\frac{\pi}{2 \Delta y}\left(y+I_{y}\right)\right)}{\tanh \left(\frac{\pi}{2 \Delta y}\left(x-\Delta x k-I_{x}\right)\right)}\right]\right. \\
\left.+\arctan \left[\frac{\tan \left(\frac{\pi}{2 \Delta y}\left(y-I_{y}\right)\right)}{\tanh \left(\frac{\pi}{2 \Delta y}\left(x-\Delta x k-I_{x}\right)\right)}\right]\right)
\end{array}
$$

and

$$
\begin{aligned}
& B_{w r}=-\mu_{0} \frac{\partial \Omega}{\partial x} \\
& B_{w \theta}=-\mu_{0} \frac{\partial \Omega}{\partial y}
\end{aligned}
$$

The solution $\left(B_{w}\right)$ can be mapped back to the original slotted air gap using the permeance function

$$
B_{s}=B_{w}\left(\frac{\partial w}{\partial s}\right)^{*}=B_{w}\left(\frac{\partial w}{\partial z} \frac{\partial z}{\partial s}\right)^{*}
$$

where

$$
\begin{aligned}
& \frac{\partial w}{\partial z}=\operatorname{evaldiff}(f, w) \\
& \frac{\partial z}{\partial s}=\frac{1}{R_{g} e^{j \theta}} .
\end{aligned}
$$

The function evaldiff is a MATLAB function introduced in its workspace by SC Toolbox.

\section{Harmonic Modelling}

The geometry of the test bench machine, shown in Fig 1, has well defined regions like slots, air gap, and rotor permanent magnets. Due to this fact, the model parameters are described by periodic functions for each region. For a $2 \mathrm{D}$ problem, using the definition of magnetic vector potential and Ampere's circuital law, the following system of differential equations can be derived in the polar coordinate system:

$$
\begin{aligned}
\mu_{r} H_{r} & =\frac{1}{r} \frac{\partial A_{z}}{\partial \theta} ; \\
\mu_{\theta} H_{\theta} & =-\frac{\partial A_{z}}{\partial r} ; \\
J_{z} & =\frac{1}{r} H_{\theta}+\frac{\partial H_{\theta}}{\partial r}-\frac{1}{r} \frac{\partial H_{r}}{\partial \theta} .
\end{aligned}
$$

where $A_{z}$ is the component of vector magnetic potential in the axial direction $(z), H_{r}$ and $H_{\theta}$ are the radial and tangential field strength respectively, $\mu_{r}, \mu_{\theta}$ and $J_{z}$ are the distribution of the iron permeability and current density, which is zero in this study. The equations (15-17) are solved for $A_{z}$,

$$
A_{z}=W r^{\lambda_{m}} a+W r^{-\lambda_{m}} b+r^{2} G_{1} .
$$

where $\lambda_{m}$ and $W$ are the results of eigen-decomposition of the root of the quadratic equation resulting from the differential equation, $G_{1}$ is the constant which contains the source components from the region, and $a$ and $b$ are the unknowns. For coupling multiple regions with each other, continuous boundary conditions are applied [5].

\section{E. Finite Element Analysis}

The results from the analytical models are compared with the results from a commercial FEA software (FLUX2D). The geometry of the model is finely meshed so that further refinement of the mesh will not improve the results. The assumptions considered in the FEA model are identical to the ones in the analytical models. The permeability of the iron is assigned to a large value to approximate infinite permeability. The same computation is repeated for different number of mesh nodes in order to study their influence on computational time.

\section{DISCUSSION ON THE RESULTS}

\section{A. Magnetic field solution}

From Fig. 3 it can be observed that the radial flux density waveforms of the analytical models, except the relative permeance model, are matching well with FEA result. The tangential field, in Fig. 4, is accurately calculated by the Harmonic and SC Toolbox models. The complex permeance model has some deviations. 


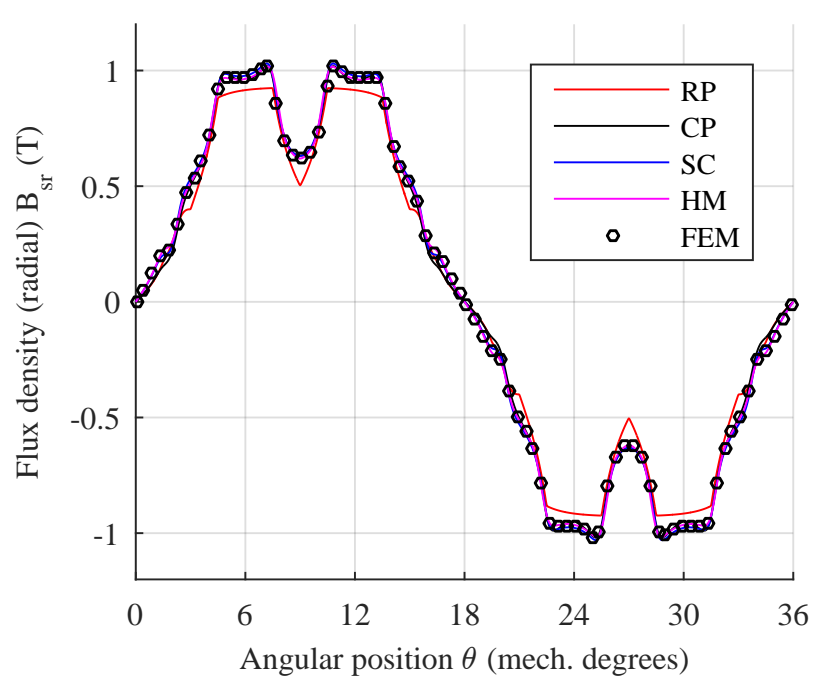

Fig. 3. Radial flux density in the air gap

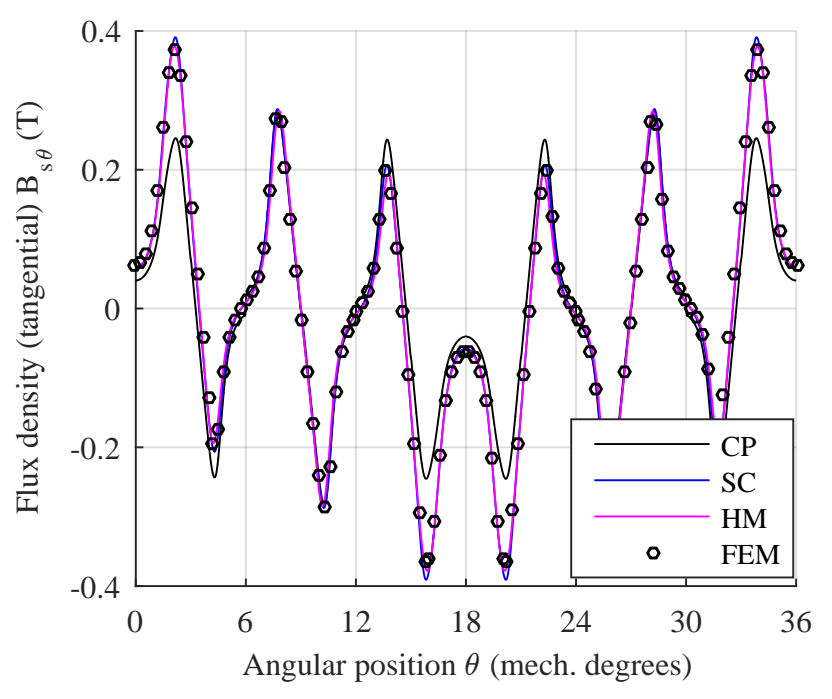

Fig. 4. Tangential flux density in the air gap

\section{B. Flux linkage}

The flux linkage of phase A winding is computed by integrating the radial flux density component across the coil pitch and multiplying it by the number of coils connected in series. The analytical models, except relative permeance model, have good correspondence with FEA model as shown in Fig.5.

\section{Cogging torque}

The cogging torque is more sensitive to the accuracy of the field solutions. A closed form solution based on Maxwell stress tensor is used for calculating the cogging torque in the complex permeance model. In harmonic and SC Toolbox models, tangential component of Maxwell stress tensor is numerically integrated. In the relative permeance model, the

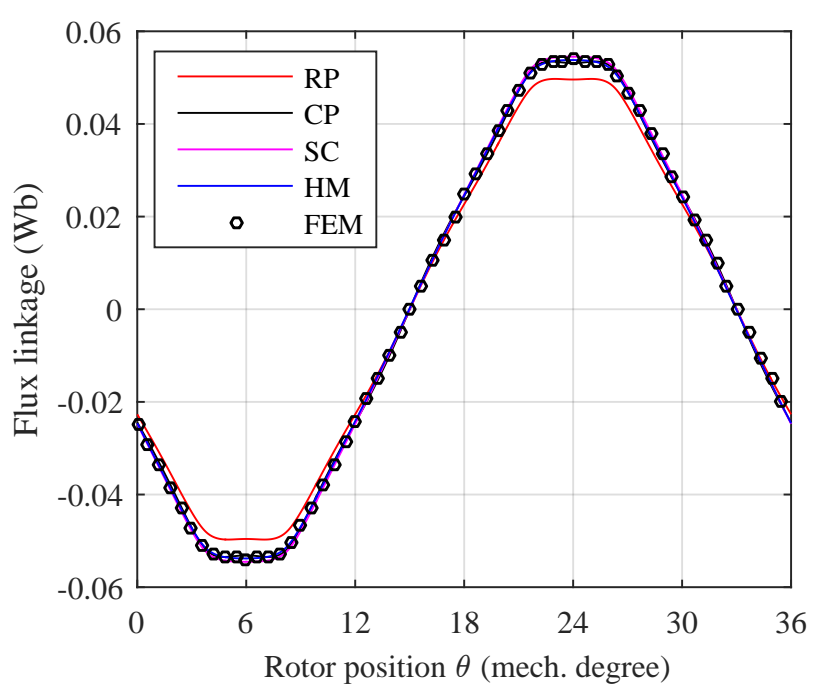

Fig. 5. Flux linkage of phase A

cogging torque is calculated by integrating the lateral forces acting on the teeth walls as in (6). The FEM software uses virtual work method for computing the forces and torque. From Fig. 6 it is evident that the field solutions from harmonic and SC Toolbox models are more accurate. Although the radial field component of the complex permeance model is accurate, the deviation in tangential component produces higher cogging torque values. Due to the inaccuracy of the radial flux density solution produced by the relative permeance model, its cogging torque values are also high.

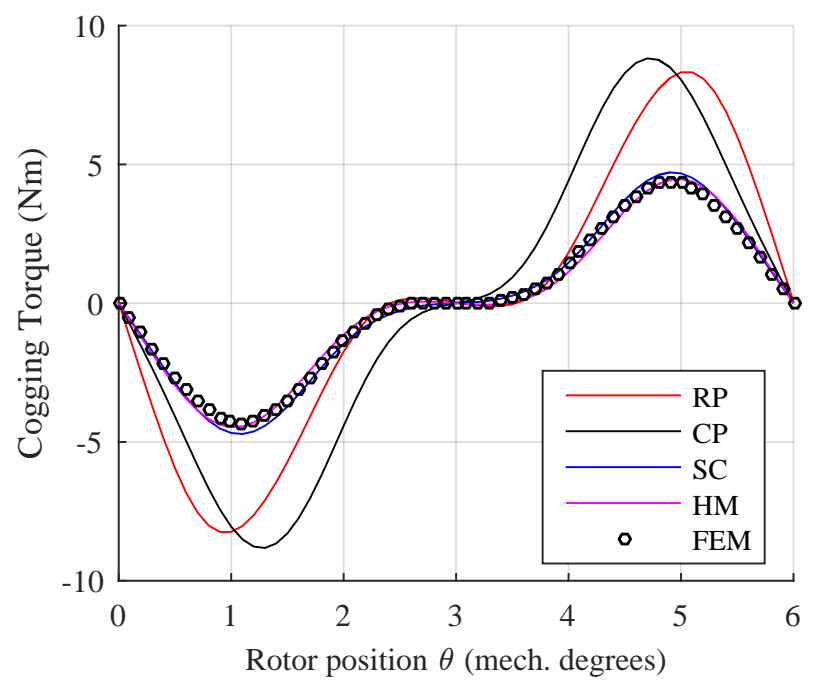

Fig. 6. Cogging torque of the given test bench machine

\section{Performance analysis}

The accuracy of the field solutions and calculation time are influenced by the number of harmonics and discretization points. The number of harmonics represents the maximum 
order of harmonics used in the slotless air gap field solution calculated using (1) to (4) and in the Fourier equation (18) that defines the parameters in the harmonic model. The number of discretization points denotes the number of evaluation points in the middle of the air gap along an angular span of two pole pitches used for calculation of the air-gap field.

A parameter sweep analysis is performed to obtain the minimum time required to get a stable result in terms of variation of RMS value of the cogging torque waveform. The reference RMS value is the one obtained using FEA with maximum number of nodes. For example, in Fig. 7 the RMS value of the cogging torque gets stabilized after 30 harmonics and the corresponding calculation time is approx. 6 seconds. The offset between the stabilized RMS value and the reference RMS value from FEM indicates the accuracy. The fastest convergence is observed for the harmonic model and its accuracy is also high.

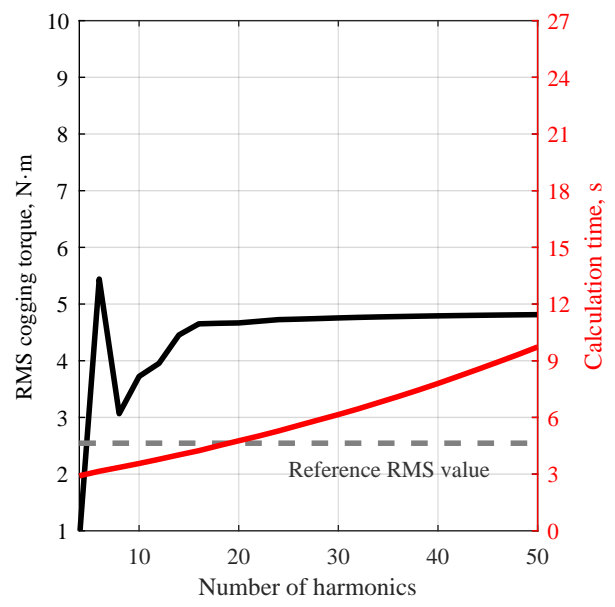

Fig. 7. Performance analysis based on number of harmonics for RP model (Discretization points $=1000)$.

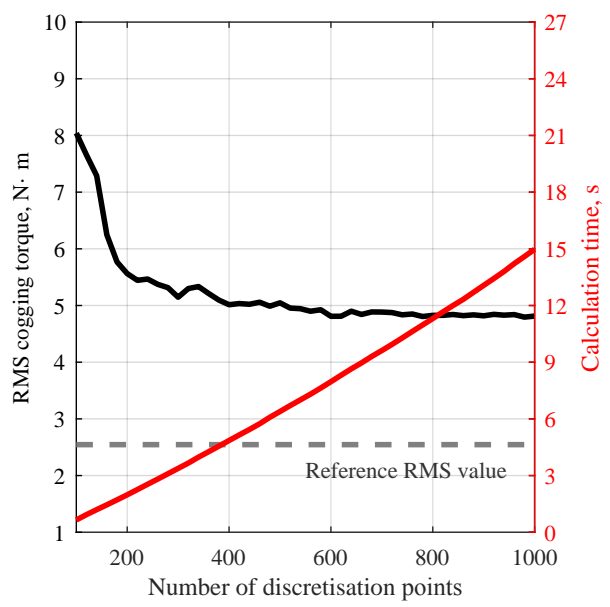

Fig. 8. Performance analysis based on number of discretization points for RP model (Harmonics $=50)$.

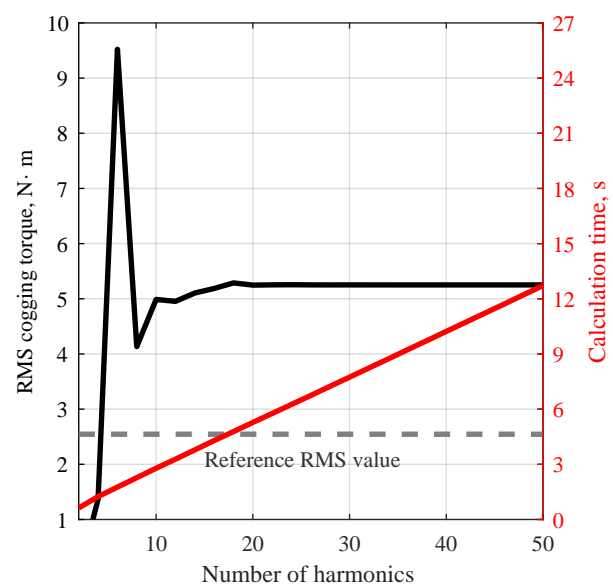

Fig. 9. Performance analysis based on number of harmonics for $\mathrm{CP}$ model (Discretization points $=50$ ).

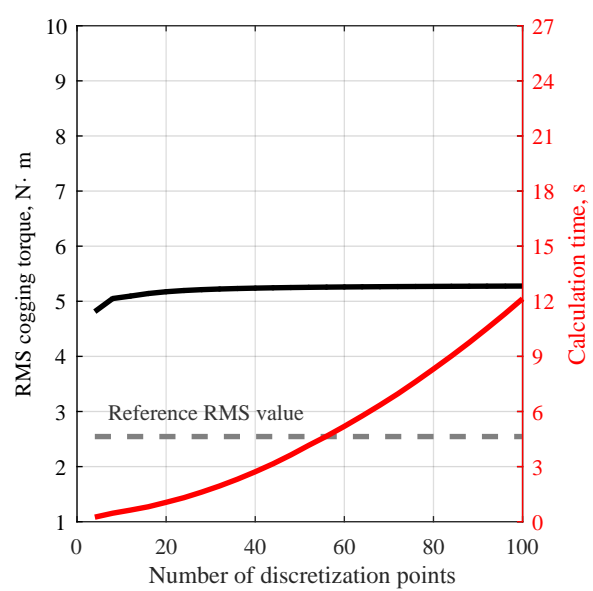

Fig. 10. Performance analysis based on number of discretization points for $\mathrm{CP}$ model $($ Harmonics $=50)$.

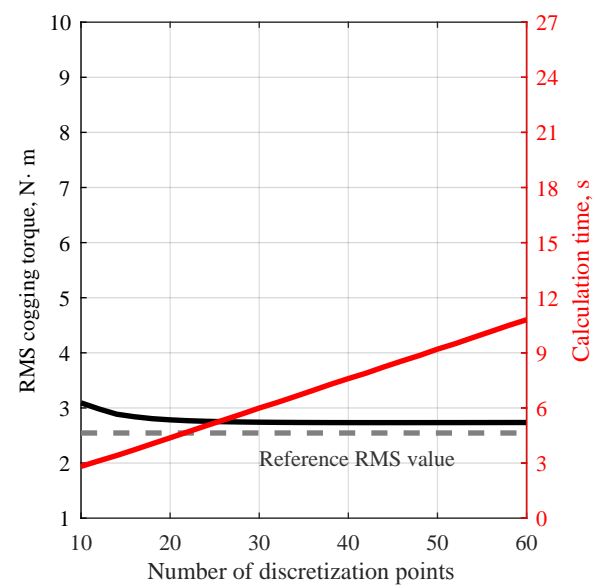

Fig. 11. Performance analysis based on number of discretization points for SC model. 


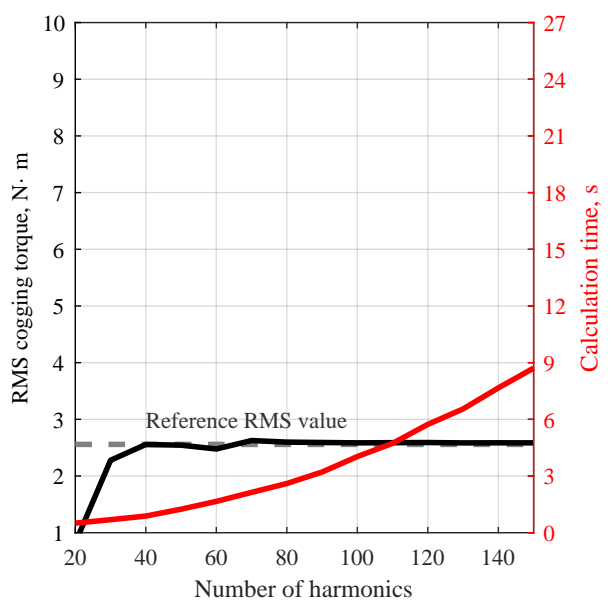

Fig. 12. Performance analysis based on number of harmonics for HM model.

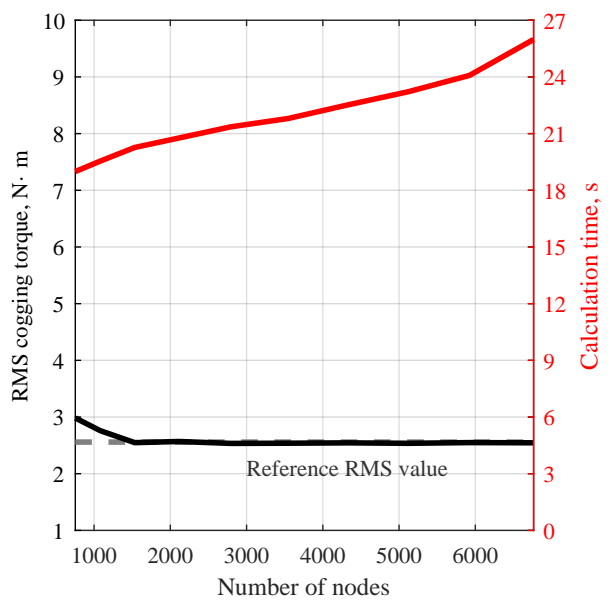

Fig. 13. Performance analysis based on number of nodes for FEM model.

\section{CONCLUSIONS}

Open circuit analysis was performed on a 20 pole surface PM machine using four different modelling techniques and the results are compared with FEA model. The accuracy of the modelling techniques are studied by comparing the cogging torque and flux linkage waveforms. Similarly, the modelling complexities are evaluated based on their computation time. The parameter sweep analysis was performed to find the minimum number of harmonics or minimum number of discretization points required to obtain a stable result in terms of RMS value of the waveform. It can be observed that the most accurate result compared to FEA is obtained by the harmonic model, which is followed by the SC Toolbox based model. The complex permeance model gives the radial component of the field solution with good accuracy, but its tangential component has some deviations. The results from the relative permeance model are not accurate enough. In terms of computation time, the harmonic model's convergence is the fastest and the complex permeance model comes next.

\section{ACKNOWLEDGMENT}

This paper is part of the ADvanced Electric Powertrain Technology (ADEPT) project which is an EU funded Marie Curie ITN project, grant number 607361 [2]. Within ADEPT, virtual and hardware tools are created to assist the design and analysis of future electric propulsions. Especially within the context of the paradigm shift from fuel powered combustion engines to alternative energy sources (e.g. fuel cells, solar cells, and batteries) in vehicles like motorbikes, cars, trucks, boats, planes. The design of these high performance, low cost and clean propulsion systems has stipulated an international cooperation of multiple disciplines such as physics, mathematics, electrical engineering, mechanical engineering and specialisms like control engineering and safety. By cooperation of these disciplines in a structured way, the ADEPT program provides a virtual research lab community from labs of European universities and industries [13].

\section{REFERENCES}

[1] J. W. Jansen, J. P. C. Smeets, T. T. Overboom, J. M. M. Rovers, and E. A. Lomonova, "Overview of analytical models for the design of linear and planar motors," Magnetics, IEEE Transactions on, vol. 50, no. 11, pp. 1-7, Nov 2014.

[2] E. A. Lomonova, J. J. H. Paulides, S. Wilkins, and J. Tegenbosch, "Adept: "advanced electric powertrain technology" - virtual and hardware platforms," in Ecological Vehicles and Renewable Energies (EVER), 2015 Tenth International Conference, March 2015, pp. 1-10.

[3] B. Hague, The principles of electromagnetism applied to electrical machines: (formerly titled: Electromagnetic problems in electrical engineering). Dover Publications, 1962.

[4] K. J. Binns, P. J. Lawrenson, and C. W. Trowbridge, The analytitcal and numerical solution of electric and magnetic fields. John Wiley \& Sons, 2001.

[5] R. L. J. Sprangers, J. J. H. Paulides, B. L. J. Gysen, and E. Lomonova, "Towards magnetic saturation in semi-analytical harmonic modeling for electric machine analysis," Magnetics, IEEE Transactions on, vol. PP, no. 99, pp. 1-1, 2015.

[6] E. P. Furlani, Electric Machines and Drives, I. Mayergoyz, Ed. Acadenic Press, 2001.

[7] Z. Zhu, D. Howe, E. Bolte, and B. Ackermann, "Instantaneous magnetic field distribution in brushless permanent magnet dc motors. i. opencircuit field," Magnetics, IEEE Transactions on, vol. 29, no. 1, pp. 124 135, 1993.

[8] D. Žarko, D. Ban, T. Lipo et al., "Analytical solution for cogging torque in surface permanent-magnet motors using conformal mapping," Magnetics, IEEE Transactions on, vol. 44, no. 1, pp. 52-65, 2008.

[9] K. Boughrara, D. Zarko, R. Ibtiouen, O. Touhami, and A. Rezzoug, "Magnetic field analysis of inset and surface-mounted permanent-magnet synchronous motors using schwarz-christoffel transformation," Magnetics, IEEE Transactions on, vol. 45, no. 8, pp. 3166-3178, 2009.

[10] B. L. J. Gysen, K. J. Meessen, J. J. H. Paulides, and E. A. Lomonova, "General formulation of the electromagnetic field distribution in machines and devices using fourier analysis," Magnetics, IEEE Transactions on, vol. 46, no. 1, pp. 39-52, Jan 2010.

[11] M. Curti, J. Paulides, and E. Lomonova, "An overview of analytical methods for magnetic field computation," in Ecological Vehicles and Renewable Energies (EVER), 2015 Tenth International Conference on, March 2015, pp. 1-7.

[12] Z. Zhu, D. Howe, and C. Chan, "Improved analytical model for predicting the magnetic field distribution in brushless permanent-magnet machines," Magnetics, IEEE Transactions on, vol. 38, no. 1, pp. 229238,2002

[13] A. Stefanskyi, A. Dziechciarz, F. Chauvicourt, G. E. Sfakianakis, K. Ramakrishnan, K. Niyomsatian, M. Curti, N. Djukic, P. Romanazzi, S. Ayat, S. Wiedemann, W. Peng, A. Tamas, and S. Stipetic, "Researchers within the EU funded Marie Curie ITN project ADEPT, grant number 607361," 2013-2017. 